\title{
Response of clamped structural slabs subjected to a dynamic point load via BEM
}

\author{
J. Antonio *, A. Tadeu, N. Simões \\ University of Coimbra, Department of Civil Engineering, 3030-290 Coimbra, Portugal
}

Received 3 June 2002; received in revised form 9 September 2002; accepted 9 September 2002

\begin{abstract}
This work computes the response of clamped slabs when subjected to spatially sinusoidal harmonic line loads via the Boundary Element Method (BEM). The formulation uses 2.5D Green's functions for the steady state response of a homogeneous threedimensional free solid layer formation of infinite extent, proposed earlier by the authors. The inclusion of these Green's functions in the BEM formulation avoids the discretization of free horizontal surfaces, which contributes to the efficiency of the BEM model. Frequency and time responses have been computed for slabs with and without lateral confinements, for different thickness and varying spatially sinusoidal harmonic line loads.
\end{abstract}

(C) 2002 Elsevier Science Ltd. All rights reserved.

Keywords: Boundary Element Method; Slab; Green's functions; free solid layer formation

\section{Introduction}

Several approaches for simulating the dynamic response of infinite and finite plates have been developed over the years. Given the large number of variables, the mathematical simulation of the phenomena involved is very complex. However, certain mathematical models have been developed that account for a restricted number of those variables, which have led to a set of simplified models. Among the simplified formulae established are expressions for obtaining the propagation of plane waves in an infinite plate and the acoustic insulation provided by such structures [1].

In a previous work [2] the authors derived a set of 2.5D Green's functions for the simulation of the wavefield produced in a homogeneous three-dimensional free solid layer formation (slab) of infinite extent, subjected to a spatially sinusoidal harmonic line load, polarized along the horizontal, vertical and $z$ directions. This problem is commonly referred to as a $2.5 \mathrm{D}$ formulation since the load is $3 \mathrm{D}$ but the geometry is $2 \mathrm{D}$, that is, it does not

\footnotetext{
* Corresponding author. Tel.: +351 239797 196; fax: +351 239 797190.

E-mail address: julieta@dec.uc.pt (J. Antonio).
}

vary along one direction. The three-dimensional problem was formulated as a summation of two-dimensional problems for varying wavenumbers along the $z$ direction, following the technique of Bouchon [3] and Kim et al. [4]. Each two-dimensional problem was calculated as a continuous superposition of plane waves with different wave numbers in the $x$ direction $\left(k_{\mathrm{n}}\right)$, adopting the approach used first by Lamb [5]. The final expressions were obtained ascribing null stresses to the two horizontal surfaces of the slab.

The present paper includes these Green's functions in a direct BEM formulation for computing the response of a slab of semi-infinite or finite extent along the $x$ direction, when subjected to a spatially sinusoidal harmonic line load along the $z$ direction. The slab is considered to be of infinite extent in the $z$ direction. As the 2.5D Green's functions simulate both the free horizontal surfaces of the slab, only the lateral boundaries need to be discretized by boundary elements. The lateral confinements of the slabs are assumed to be clamped, meaning that null displacements are ascribed along these edges.

The computations are first performed in the frequency domain for different spatial wavenumbers in the $z$ dimension. Time responses are then obtained by means of inverse Fourier transforms. Complex frequencies are used to avoid aliasing phenomena [6]. 
This paper first presents the BEM formulation. Then the BEM model is validated using a direct boundary element method requiring the full discretization of all the boundaries (using Green's functions for an unbounded space). Finally, the 2.5D Green's functions and the present BEM model are applied to simulate the threedimensional wave propagation within slabs of unlimited and limited extent.

\section{BEM formulation}

Let us assume the existence of a two-dimensional solid elastic slab with free (null tensions) horizontal boundaries and rigid lateral boundaries (clamped). The Boundary Element Method (BEM) is applied in the frequency domain $(\omega)$ to compute the 3D field generated by a spatially sinusoidal harmonic line load buried in such a structure. As the BEM formulation used here incorporates the Green's functions derived in an earlier work [2], taking the presence of the horizontal free boundaries into account, the discretization with boundary elements is limited to the lateral boundaries.

The essential BEM equations are widely known [7,8], and therefore they are only briefly described here. The boundary integral equations, in the absence of distributed loads and in the presence of virtual point loads, $\delta(x-$ $\boldsymbol{x}_{0}$ ), lead to the following equation,

$$
\begin{gathered}
c_{\mathrm{ij}} u_{\mathrm{j}}\left(\boldsymbol{x}_{0}, \omega\right)=\int_{\mathrm{C}} t_{\mathrm{i}}(\boldsymbol{x}, \boldsymbol{v}, \omega) G_{\mathrm{ij}}\left(\boldsymbol{x}, \boldsymbol{x}_{0}, \omega\right) d s \\
-\int_{\mathrm{C}} H_{\mathrm{ij}}\left(\boldsymbol{x}, \boldsymbol{v}, \boldsymbol{x}_{0}, \omega\right) u_{\mathrm{j}}(\boldsymbol{x}, \omega) d s
\end{gathered}
$$

where, $i, j=1,2$ refers to the normal and tangential directions in relation to the boundary surface; $i, j=$

3 indicates the $\mathrm{z}$ direction; $G_{\mathrm{ij}}\left(\boldsymbol{x}, \boldsymbol{x}_{0}, \omega\right), H_{\mathrm{ij}}\left(\boldsymbol{x}, \boldsymbol{v}, \boldsymbol{x}_{0}, \omega\right)$ are the displacements and tractions in direction $j$ at $\boldsymbol{x}$, on boundary $C$, provoked by a unit sinusoidal line load acting at source, $x_{\mathbf{0}}$, in direction $i$; vector $v$ is the unit outward normal at the boundary, and $c_{\mathrm{ij}}$ is a constant that equals $\delta_{\mathrm{ij}} / 2$ for a smooth boundary, where Kronecker's delta is represented by $\delta_{\mathrm{ij}}$. The $2.5 \mathrm{D}$ Green's functions $G_{\mathrm{ij}}$ are not given here, since they can be found in Tadeu and António [2] as $G_{\mathrm{ij}}^{\text {slab }}$, where the steady-state response of a spatially sinusoidal harmonic line load on a homogeneous three-dimensional slab, has been computed.

The imposition of the lateral boundary conditions (null displacements) leads to a simplified form of Eq. (1):

$$
\int_{\mathrm{C}} t_{\mathrm{i}}(\boldsymbol{x}, \boldsymbol{v}, \omega) G_{\mathrm{ij}}\left(\boldsymbol{x}, \boldsymbol{x}_{0}, \omega\right) d s=0
$$

The displacement field is transformed, at each element, into the normal, tangential and local $(z)$ co-ordinate system, from equilibrium relations. When the boundary is discretized into $N$ boundary elements and the nodal displacements within each element are assumed both to be constant and to have the same value at the respective nodal point, Eq. (2) becomes,

$$
\sum_{\mathrm{n}=1}^{\mathrm{N}} G_{\mathrm{ij}}^{\mathrm{kn}} t_{\mathrm{j}}^{\mathrm{n}}=0
$$

In Eq. (3), the element number at the point where the virtual load is applied is defined by $k$, the boundary values in element $n$ are identified by $t_{\mathrm{j}}^{\mathrm{n}}$, and $G_{\mathrm{ij}}^{\mathrm{kn}}$ represents the element integrals

$G_{\mathrm{ij}}^{\mathrm{kn}}=\int_{\mathrm{C}_{\mathrm{n}}} G_{\mathrm{ij}}\left(\boldsymbol{x}, \boldsymbol{x}_{0}, \omega\right) d s$

where $C_{\mathrm{n}}$ is the boundary segment.

The application of a virtual load on the boundary, in each node, leads to a system of linear equations relating nodal forces and nodal displacements. These equations can be solved for the nodal tractions.

In the presence of an incident wave within the slab Eq. (2) is modified as follows,

$\int_{\mathrm{C}} t_{\mathrm{i}}(\boldsymbol{x}, \boldsymbol{v}, \omega) G_{\mathrm{ij}}\left(\boldsymbol{x}, \boldsymbol{x}_{0}, \omega\right) d s+u_{\mathrm{j}}^{\mathrm{inc}}\left(\boldsymbol{x}_{0}, \omega\right)=0$

where $u_{j}^{\text {inc }}\left(x_{0}, \omega\right)$ are the displacements originated by the incident wave. In the above equation the incident field is obtained analytically as the sum of the source terms (two-and-a-half dimensional full-space) and the surface terms resulting from the two horizontal surfaces (see Tadeu and António [2]). A system of equations can be established from the integral equations subjected to the boundary conditions, and discretized appropriately.

When the element to be integrated is not the loaded element, the required integrations in Eq. (5) are accomplished using Gaussian quadrature. For the loaded element, the existing singular integrands in the source terms of the Green's functions are calculated in closed form $[9,10]$, while the integrands involving the surface terms originated on both free and rigid surfaces are solved using a Gaussian quadrature scheme.

Displacements in the time domain are obtained applying a numerical inverse fast Fourier transform to the frequency responses. The time responses are obtained for a source modeled as a Ricker wavelet [11] with a specific characteristic frequency. The Fourier transformations are obtained by discrete summations over frequencies, which is mathematically equivalent to adding periodic sources at temporal intervals $T=2 \pi / \Delta \omega$, where $\Delta \omega$ represents the frequency steps. Complex frequencies are 
used, in the form $\omega_{\mathrm{c}}=\omega-i \eta$ (with $\eta=0.7 \Delta \omega$ ), to avoid aliasing phenomena. In the time domain, this effect is removed by rescaling the response using an exponential window $e^{\eta \mathrm{t}}[12]$.

\section{BEM validation}

The BEM model is validated by comparing the results with those given by a BEM formulation which needs the full discretization of the boundaries. A solid layer $10.0 \mathrm{~m}$ thick and $20.0 \mathrm{~m}$ wide is used to validate the response (see Fig. 1a). A spatially sinusoidal harmonic line load $\left(k_{z}=0.4 \mathrm{rad} / \mathrm{m}\right)$ is applied at $\left(x_{0}=-4.0 \mathrm{~m}\right.$, $y_{0}=3.0 \mathrm{~m}$ ), acting along the direction $y$. The elastic material of the formation, with a density of $\rho=$ $2140 \mathrm{~kg} / \mathrm{m}^{3}$, allows a dilatational and a shear wave velocity of $\alpha=4208 \mathrm{~m} / \mathrm{s}$ and $\beta=2656 \mathrm{~m} / \mathrm{s}$, respectively. The responses are calculated in the frequency range $[2.50,320.0 \mathrm{~Hz}]$ with a frequency increment of $2.5 \mathrm{~Hz}$. The surface scattered displacement field in the $i$ direction due to a load acting along $y, G_{\mathrm{iy}}^{\text {surf }}$ is computed at a receiver point placed at $x=-6.0 \mathrm{~m}$ and $y=$ $6.0 \mathrm{~m}$.

Fig. 1(b-d) presents the computed responses. The solid lines represent the present BEM formulation solutions, while the marked points correspond to the BEM solution using the full boundary discretization. The square marks refer to the real part of the response while the round marks represent the imaginary part. A very good agreement between the two solutions can be observed.

\section{Applications}

The BEM model described here is used to study the wave propagation generated by a harmonic sinusoidal line load acting along the vertical direction at $\left(x_{0}, y_{0}\right)$ within a concrete $(\alpha=3498.6 \mathrm{~m} / \mathrm{s}, \beta=2245 \mathrm{~m} / \mathrm{s}$, and $\rho=2500 \mathrm{Kg} / \mathrm{m}^{3}$ ) slab. Three different configurations are analyzed: a slab of infinite extent; a slab of semiinfinite extent with one lateral rigid boundary; and a slab of finite extent with two lateral rigid boundaries (see Fig. 2).

The geometry of the models is constant along the $z$ direction, which allows the 3D solution to be obtained by adding together a series of two-dimensional problems, for the varying effective wavenumbers, $k_{\alpha}=$ $\sqrt{k_{\mathrm{p}}^{2}-k_{\mathrm{z}}^{2}}$ with $k_{\mathrm{p}}=\omega / \alpha$ and $k_{\beta}=\sqrt{k_{\mathrm{s}}^{2}-k_{\mathrm{z}}^{2}}$ with $k_{\mathrm{s}}=$ $\omega / \beta$ [2], where $k_{\mathrm{z}}$ is the axial wavenumber, once a Fourier transformation has been applied to the problem in the $z$ direction. In this $k_{\mathrm{z}}$ domain, the system is struck by a sinusoidal line load acting along the vertical direction $(y)$. In the frequency domain, the incident displacement field originated by this load is given by

$G_{\mathrm{xy}}^{\mathrm{full}}=\frac{\mathrm{i}}{4 \rho \omega^{2}}\left(\frac{x-x_{0}}{r}\right)\left(\frac{y-y_{0}}{r}\right) B_{2}$ (a)
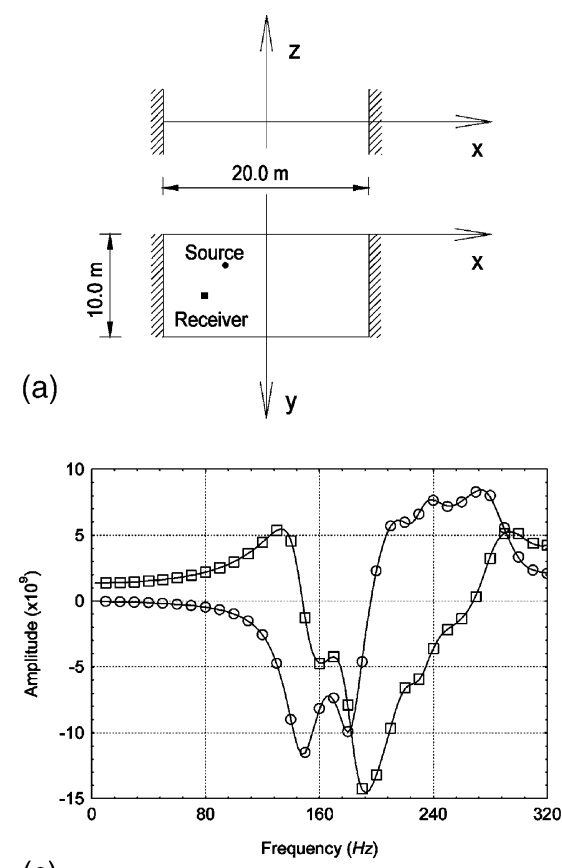

(c)
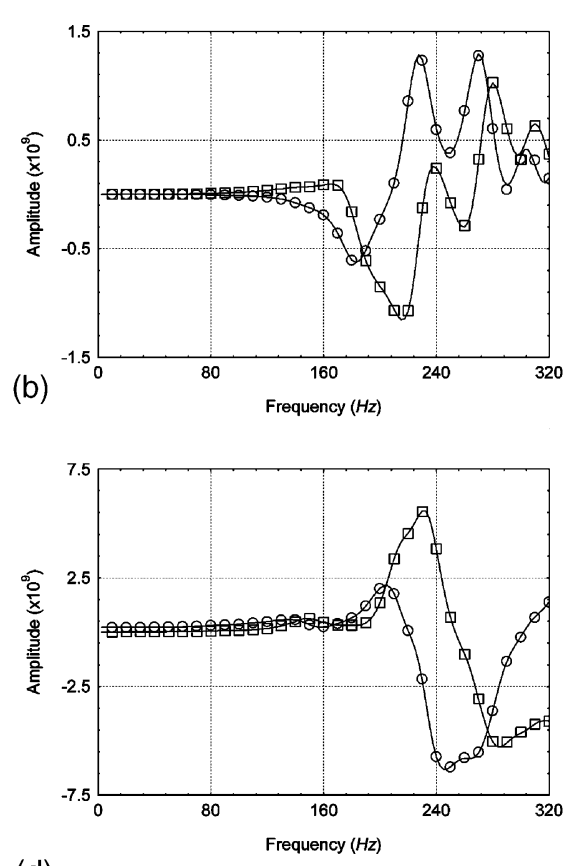

(d)

Fig. 1. Spatially sinusoidal harmonic line load along the $z$ direction in a slab, applied in the $y$ direction: (a) Geometry of the problem; (b) $G_{\mathrm{xy}}^{\text {surf }}$ solutions; (c) $G_{y y}^{\text {surf }}$ solutions; (d) $G_{z y}^{\text {surf }}$ solutions. 

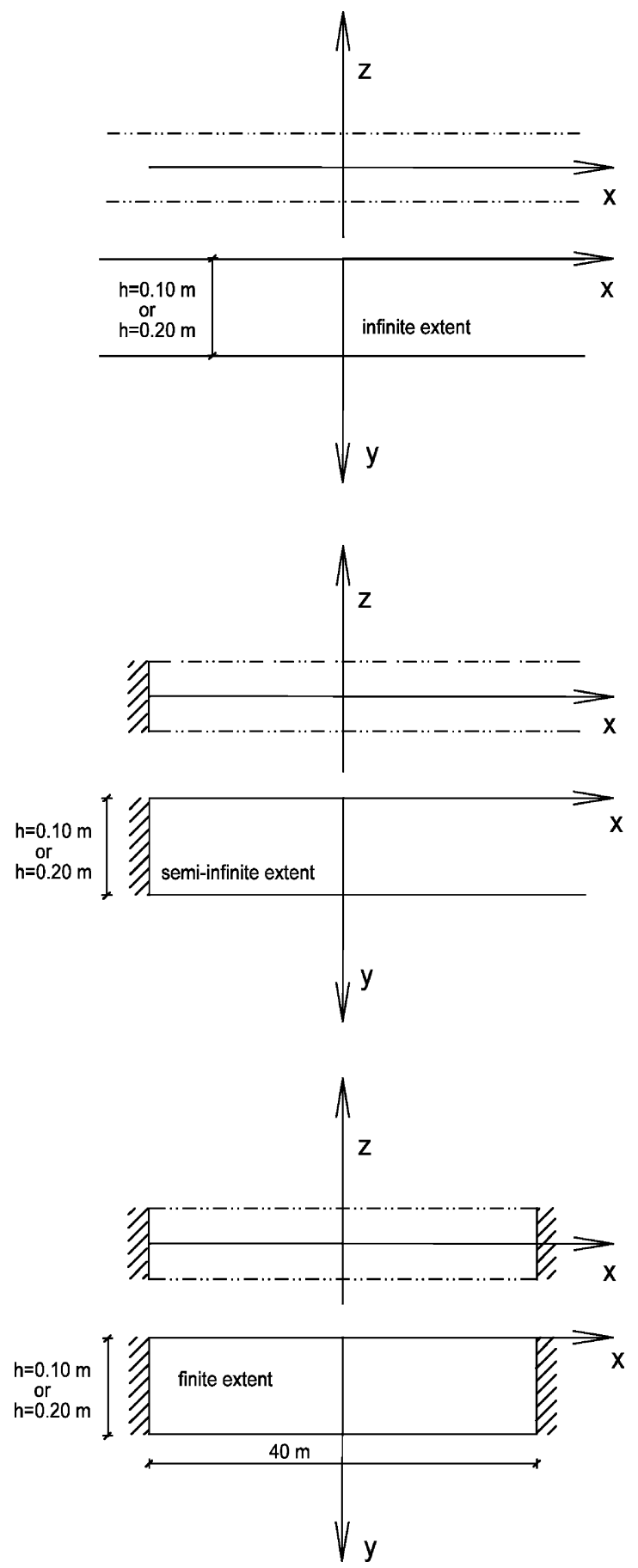

Fig. 2. Geometry of the models.

$G_{\mathrm{yy}}^{\mathrm{full}}=\frac{\mathrm{i}}{4 \rho \omega^{2}}\left[k_{\mathrm{s}}^{2} H_{0 \beta}-\frac{1}{r} B_{1}+\left(\frac{y-y_{0}}{r}\right)^{2} B_{2}\right]$

$G_{\mathrm{zy}}^{\mathrm{full}}=\frac{-k_{\mathrm{z}}}{4 \rho \omega^{2}}\left(\frac{y-y_{0}}{r}\right) B_{1}$

with

$r=\sqrt{\left(x-x_{0}\right)^{2}+\left(y-y_{0}\right)^{2}}$

$H_{\mathrm{n} \alpha}=H_{\mathrm{n}}^{(2)}\left(k_{\alpha} r\right)$
$H_{\mathrm{n} \beta}=H_{\mathrm{n}}^{(2)}\left(k_{\beta} r\right)$

$B_{\mathrm{n}}=k_{\beta}^{\mathrm{n}} H_{\mathrm{n} \beta}-k_{\alpha}^{\mathrm{n}} H_{\mathrm{n} \alpha}$

$\mathrm{i}=\sqrt{-1}$

$H_{\mathrm{n}}^{(2)}$ are the second Hankel functions of order $n$. The index $i$ in the above equations $G_{\mathrm{ij}}^{\text {full }}$, indicates the direction of the displacement while the second index, $j$, defines the direction in which the virtual load is acting.

The calculations are performed in the frequency domain from 10.0 to $10240 \mathrm{~Hz}$, with a frequency increment of $10.0 \mathrm{~Hz}$, which determines the total duration $(T=0.1 \mathrm{~s})$ of the analysis in the time domain. The time domain responses were obtained by means of an inverse Fourier transform applied to the frequency responses. Solutions in the time domain are obtained simulating a source with a Ricker time dependence, with a characteristic frequency of 1000.0 and $3000.0 \mathrm{~Hz}$. The displacements produced by a vertical line load $\left(k_{\mathrm{z}}=\right.$ $0 \mathrm{rad} / \mathrm{m}$ ) or by a vertical load with spatial sinusoidal variation along the $z$ direction $\left(k_{\mathrm{z}} \neq 0 \mathrm{rad} / \mathrm{m}\right)$, placed $0.5 \mathrm{~m}$ below the surface, are recorded at receivers, $0.01 \mathrm{~m}$ below the surface, positioned as in Fig. 3 .

\subsection{Slab of infinite extent}

Next, the results obtained when the slab is of infinite extent are analyzed. These solutions are computed directly by the $2.5 \mathrm{D}$ analytical functions that are used as Green's functions [2] in the BEM model described above. The influence of slab thickness on the wave propagation within the slab is analyzed simulating the response of slabs 0.10 or $0.20 \mathrm{~m}$ thick.

Fig. 4 shows the analytical vertical displacements when the slab is $0.10 \mathrm{~m}$ thick, in the presence of a line load $\left(k_{\mathrm{z}}=0 \mathrm{rad} / \mathrm{m}\right)$. Fig. 4a displays the frequency domain versus $k_{\mathrm{n}}$ wave number response $[10,4000 \mathrm{~Hz}$ ], while Fig. $4 \mathrm{~b}$ and $\mathrm{c}$ gives the time solution for incident pulses with characteristic frequencies of 3000 and $1000 \mathrm{~Hz}$, respectively. The frequency domain versus $k_{\mathrm{n}}$ wave number response reveals the existence of two different types of waves: body waves traveling with the shear wave velocity of $\beta=2245 \mathrm{~m} / \mathrm{s}$ (labeled S) and large amplitude dispersive guided waves. The guided waves exhibit velocities below the shear wave velocity at low frequencies and approach the shear wave velocity as the frequency increases.

The propagation of plane waves along a panel is frequently analyzed, ignoring the effects of shear and rotary inertia. The mathematical development of these assumptions leads to the following dispersion relation [1],

$c_{\mathrm{L}}=\left(\frac{D \omega^{2}}{\rho h}\right)^{0.25}$

where $c_{\mathrm{L}}$ is the phase velocity of the propagating plane 


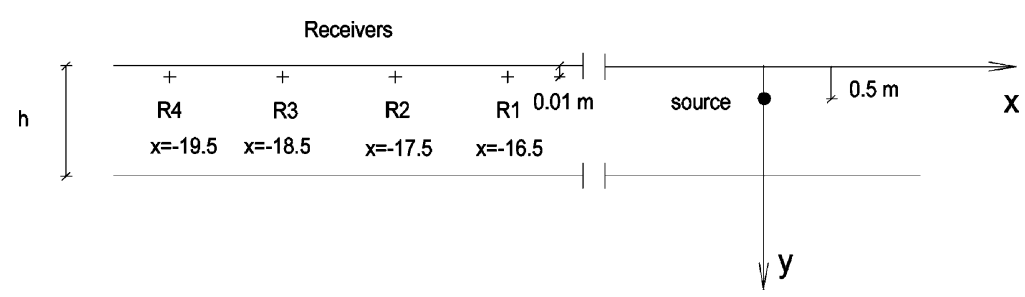

Fig. 3. Position of the source and receivers.

(a)

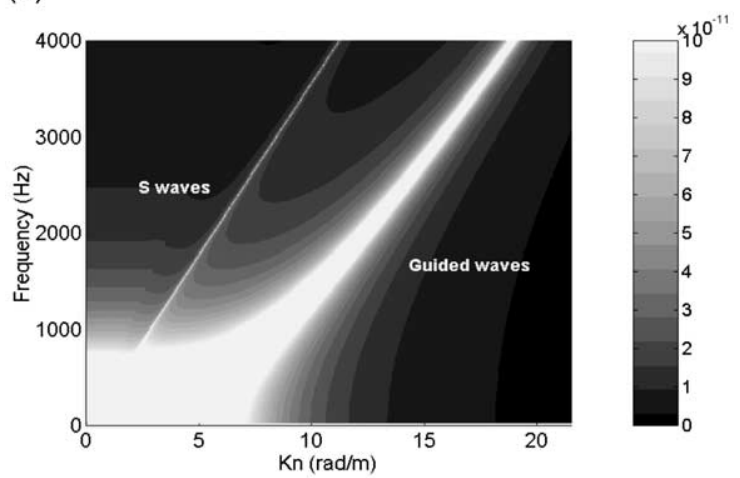

(b)

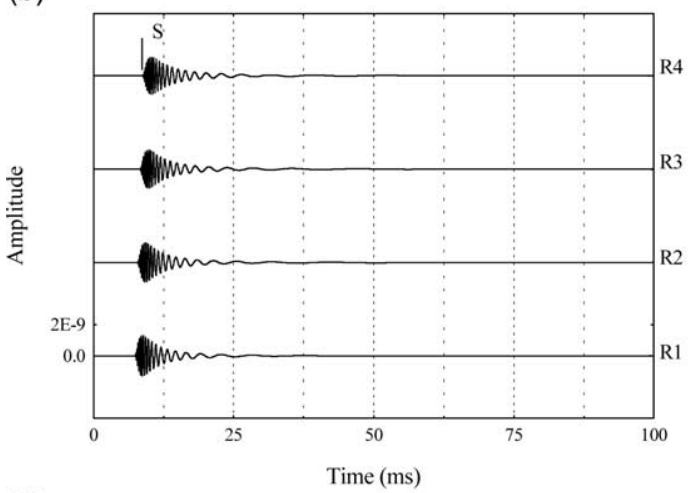

(c)

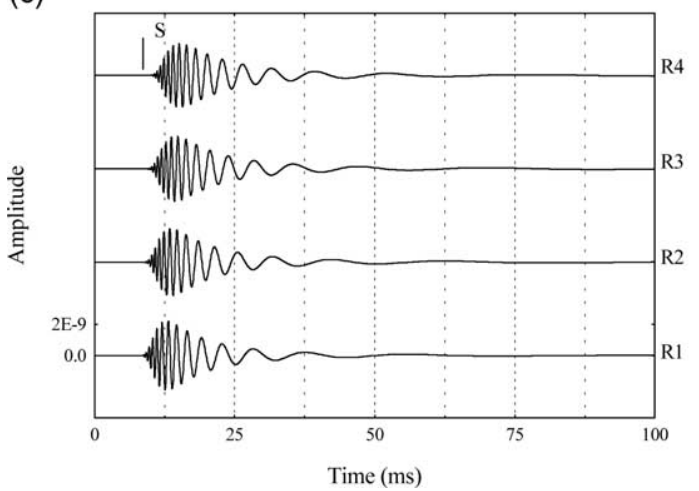

Fig. 4. Analytical vertical displacements in a slab of infinite extent and $0.10 \mathrm{~m}$ thick, for $k_{\mathrm{z}}=0 \mathrm{rad} / \mathrm{m}$ : (a) Frequency domain versus $k_{\mathrm{n}}$ wave number responses; (b) Time domain responses for a source with a characteristic frequency of $3000 \mathrm{~Hz}$; (c) Time domain responses for a source with a characteristic frequency of $1000 \mathrm{~Hz}$. waves along the plate, $\rho$ is the density of the material $\left(\mathrm{Kg} / \mathrm{m}^{3}\right), h$ is the thickness of the panel $(\mathrm{m}), \omega=2 \pi f$, $D=\frac{h^{3} E}{12\left(1-v^{2}\right)}$ with $E$ and $v$, being the Young's modulus and the Poisson's ratio, respectively. It should be noted that this equation predicts unbounded wave velocity for very short wavelength, high frequency conditions, as a consequence of the imperfect mathematical model in which effects of shear and rotary inertia have been ignored. It happens in practical applied acoustics that when the wavelength of sound air projected on a plate equals the wavelength of these bending waves, the movement of the panel increases, leading to a low sound insulation.

The presence of body waves traveling with the velocity of the $\mathrm{P}$ waves $(\alpha=3498.6 \mathrm{~m} / \mathrm{s})$ is not detectable in Fig. 4. The responses in the time domain agree with the behavior observed in the frequency plot. The arrival of a small pulse can be seen in the time responses, corresponding to waves traveling with the shear velocity, and labeled with "S". A pack of high frequency pulses is visible after the arrival of the shear waves, followed by a ring of low frequency waves. The comparison of the time responses simulated with characteristic excitation frequencies of 3000 and $1000 \mathrm{~Hz}$, shows that the guided waves in the presence of a source with lower characteristic frequency arrive at later times, owing to the absence of the high frequency components, which exhibited faster velocities. Furthermore, the amplitude of the guided waves becomes larger as the frequency of excitation decreases.

Fig. 5 shows the vertical displacements registered when the thickness of the slab is $0.20 \mathrm{~m}$, while the line load is kept at the same position. The frequency domain versus $k_{\mathrm{n}}$ wave number responses (Fig. 5a) reveals that the guided waves are less dispersive than before, particularly at low frequencies. The velocity of these waves is found to approach the shear wave velocity at frequencies below those found for a thinner slab. This effect is also visible in the time plots (Fig. 5b-c), where the guided waves are seen to be faster than those observed for a slab $0.10 \mathrm{~m}$ thick. When the characteristic frequency of the excitation source is $1000 \mathrm{~Hz}$, the guided waves are found to arrive earlier when the slab is thicker. The $S$ 
(a)

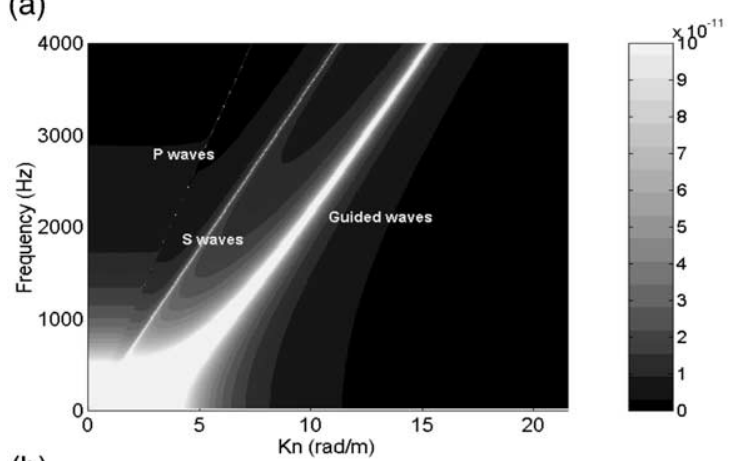

(b)

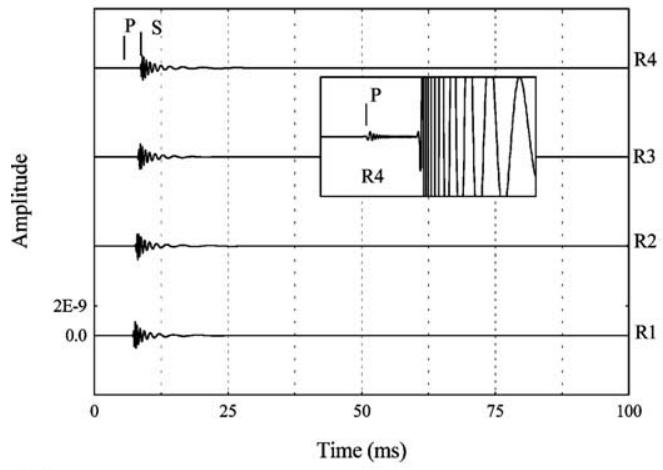

(c)

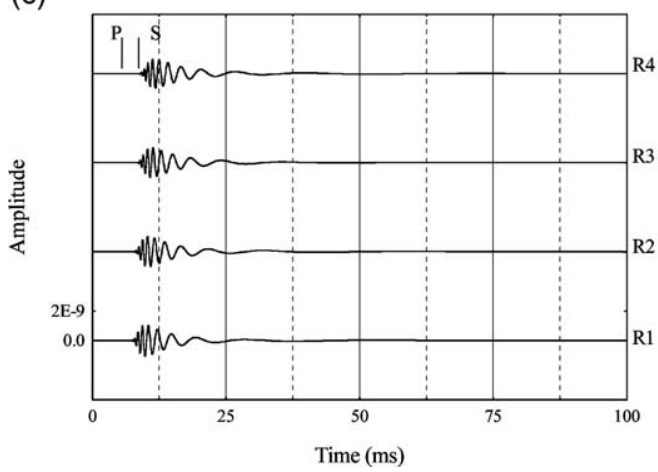

Fig. 5. Analytical vertical displacements in a slab of infinite extent, $0.20 \mathrm{~m}$ thick for $k_{\mathrm{z}}=0 \mathrm{rad} / \mathrm{m}$ : (a) Frequency domain versus $k_{\mathrm{n}}$ wave number responses; (b) Time domain responses for a source with a characteristic frequency of $3000 \mathrm{~Hz}$; (c) Time domain responses for a source with a characteristic frequency of $1000 \mathrm{~Hz}$.

body waves are still noticeable in the frequency domain versus $k_{\mathrm{n}}$ wave number responses. In addition, the $\mathrm{P}$ body waves are now visible in this figure. Given the scale of the time plots, the arrival of these waves cannot be seen clearly. Thus, the response obtained at receiver $\mathrm{R} 4(x=-19.5 \mathrm{~m})$ has been zoomed and included in Fig. 5b. In general, the amplitude of the displacements appears to decrease for the thicker slab.

The slab $0.10 \mathrm{~m}$ thick is subjected next to the incidence of a vertical load with an apparent wave velocity $c=2600 \mathrm{~m} / \mathrm{s}$, which corresponds to waves arriving at the $z$ axis with a path inclination given by $\arccos (\mathrm{v} / c)$, where $\mathrm{v}$ is the true wave velocity $\left(k_{z}=\frac{\omega}{2600} \mathrm{rad} / \mathrm{m}\right)$.
Fig. 6 displays the computed responses. The frequency domain versus $k_{\mathrm{n}}$ wave number domain (Fig. 6a) exhibits features similar to the ones observed in Fig. 4a, but appearing to be caused by waves traveling at higher velocity. This is because the direction of the wave propagation is inclined in relation to the $z$ axis, and so the path length to a receiver is shorter than when the waves propagate normally to the plane of the slab. This phenomenon is thus perceived in the frequency domain as if the waves are faster. In the time domain, it can be observed that the pulses appear earlier (see Fig. $6 \mathrm{~b}$ and $c$ and Fig. $4 b$ and $c)$.
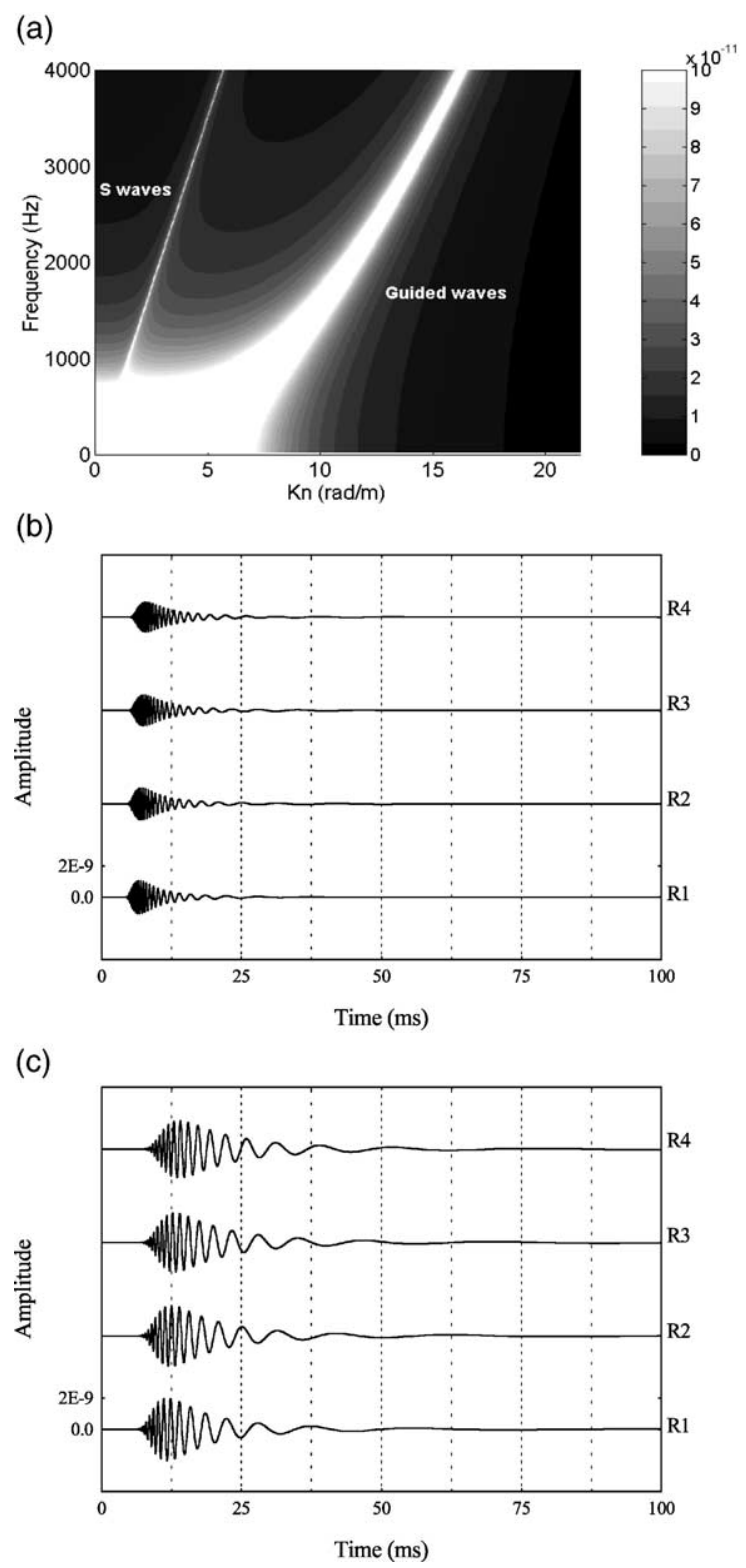

Fig. 6. Analytical vertical displacements in a slab of infinite extent, $0.10 \mathrm{~m}$ thick for an apparent velocity $c=2600 \mathrm{~m} / \mathrm{s}$ : (a) Frequency domain versus $k_{\mathrm{n}}$ wave number responses; (b) Time domain responses for a source with a characteristic frequency of $3000 \mathrm{~Hz}$; (c) Time domain responses for a source with a characteristic frequency of $1000 \mathrm{~Hz}$. 


\subsection{Confined Slab}

The slab is assumed to have one or two fixed vertical lateral confinements. As mentioned above, the free surfaces of the slab are not discretized. The lateral boundaries are modeled with a number of boundary elements, defined according to the excitation frequency of the harmonic source. The ratio between the wavelength of the incident waves and the length of the boundary elements is kept to a minimum of 15 . In no case, however, is the number of the boundary elements used to model each lateral surface less than 5 .

Fig. 7 shows synthetic time responses generated by a vertical line load $\left(k_{\mathrm{z}}=0 \mathrm{rad} / \mathrm{m}\right)$ in the presence of a slab, $0.10 \mathrm{~m}$ thick, and with a lateral confinement at $x=-20.0 \mathrm{~m}$. Fig. 7a displays the solution when the characteristic frequency of excitation is $3000 \mathrm{~Hz}$. The comparison of these results with those presented in Fig. $4 \mathrm{~b}$ reveals pronounced differences. The set of guided waves strikes the lateral rigid boundary, is reflected back, and travels in the opposite direction. The time domain exhibits two packs of high frequency pulses; given the small distance between the receivers and the lateral edge of the slab, they do not fully separate. The
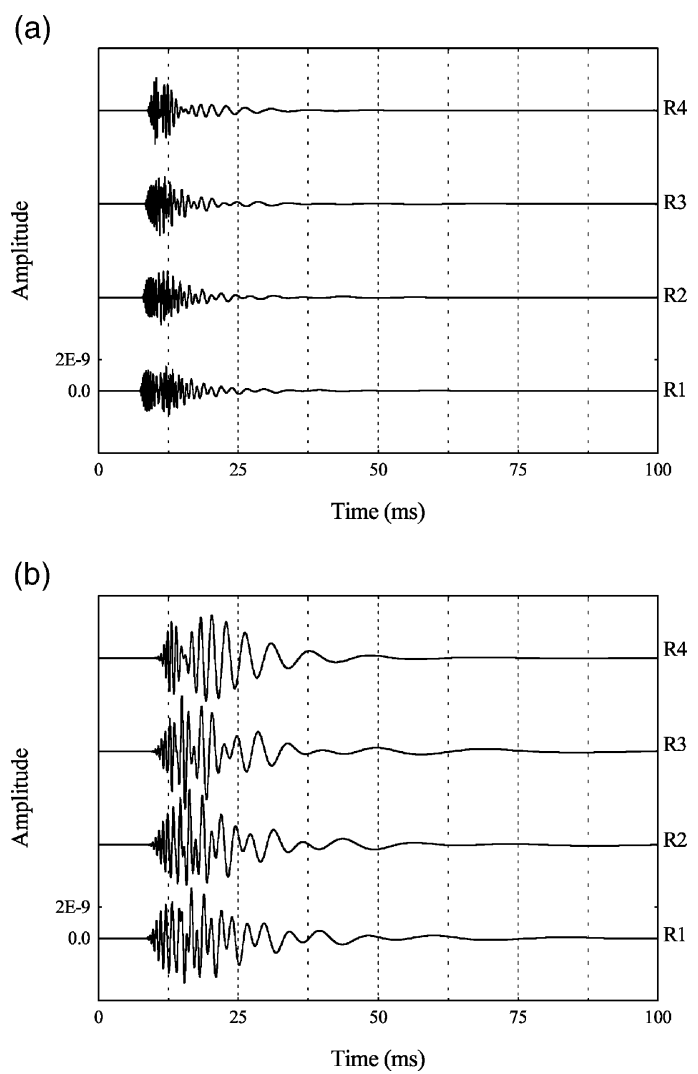

Fig. 7. Vertical time domain displacements obtained with the BEM model for the slab $0.10 \mathrm{~m}$ thick, with one lateral confinement, when a vertical line load is applied $\left(k_{z}=0 \mathrm{rad} / \mathrm{m}\right)$ : (a) Characteristic frequency of excitation $3000 \mathrm{~Hz}$; (b) Characteristic frequency of excitation $1000 \mathrm{~Hz}$. superposition of these two sets of surface waves determines that the response amplitude is enhanced mainly in the vicinity of the lateral confinement. For the receiver placed further away from the lateral edge of the slab, these two sets of pulses are seen separately in the time domain, with similar amplitudes. Fig. $7 \mathrm{~b}$ plots the response when the excitation frequency is $1000 \mathrm{~Hz}$. With the larger wavelengths, the existence of the two packs of guided pulses is not observable, given that they travel more slowly, determining their overlap. The final amplitude of the signal is consequently larger than that registered in the absence of a lateral confinement (Fig. $4 c)$. It can further be seen that the response tends to maintain an enhanced amplitude for a longer time.

Fig. 8 shows the time responses generated by a vertical line load with an apparent wave velocity $c=$ $2600 \mathrm{~m} / \mathrm{s}$ along the $z$ axis $\left(k_{\mathrm{z}} \neq 0 \mathrm{rad} / \mathrm{m}\right)$, placed within the same slab. As before, when the apparent wave velocity is smaller, the different wave pulses reach the receivers earlier. This is also seen when the present results are compared with those obtained when $k_{\mathrm{z}}=$ $0 \mathrm{rad} / \mathrm{m}$ (compare Figs. 7 and 8). Comparing the present results with those registered when the excitation load is two-dimensional, $k_{\mathrm{z}}=0 \mathrm{rad} / \mathrm{m}$, it is observed

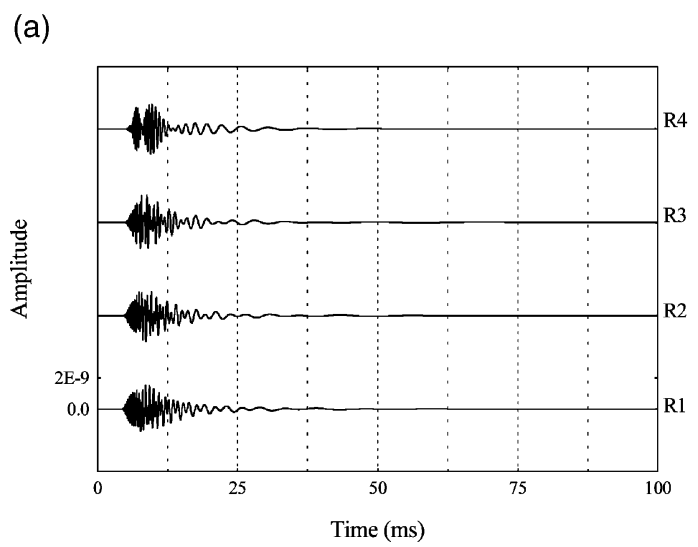

(b)

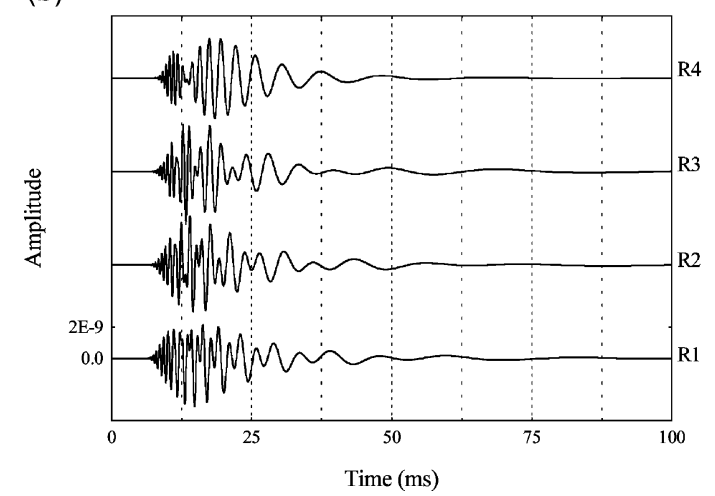

Fig. 8. Vertical time domain displacements obtained with the BEM model for the slab $0.10 \mathrm{~m}$ thick, with one lateral confinement, in the presence of a vertical load with an apparent wave velocity $\mathrm{c}=$ $2600 \mathrm{~m} / \mathrm{s}$ : (a) Characteristic frequency of excitation $3000 \mathrm{~Hz}$; (b) Characteristic frequency of excitation $1000 \mathrm{~Hz}$. 
that the amplitude decreases. It happens that in this case the scattering energy is not totally limited to the vertical $x, y$ plane containing the source, because part of this energy in fact propagates along the $z$ direction.

Fig. 9 gives the time responses when a vertical line load $\left(k_{\mathrm{z}}=0\right)$ is applied in a slab $0.10 \mathrm{~m}$ thick, with two lateral confinements placed at $x=-20.0$ and $x=$ $20.0 \mathrm{~m}$. Fig. 9a illustrates the vertical displacements when the characteristic excitation frequency is $3000 \mathrm{~Hz}$. Wave features similar to those found in the case of one lateral confinement are registered. Additional guided wave pulse trains are observable, caused by multi-reflections on both lateral confinements. After the arrival of the first incident pulse train and the pulse train first reflected on the left confinement, a second reflection coming from the right lateral confinement is visible. Then, the response becomes very complex due to the superposition of the different wave trains originated by the repetition of these scattering phenomena. The low frequency components of the response lose importance in the presence of the high frequency components contained in the multi reflection pulses. As time elapses, the amplitude of the signal tends to decrease slightly because of the energy dissipation. Fig. 9b displays the vertical

(a)

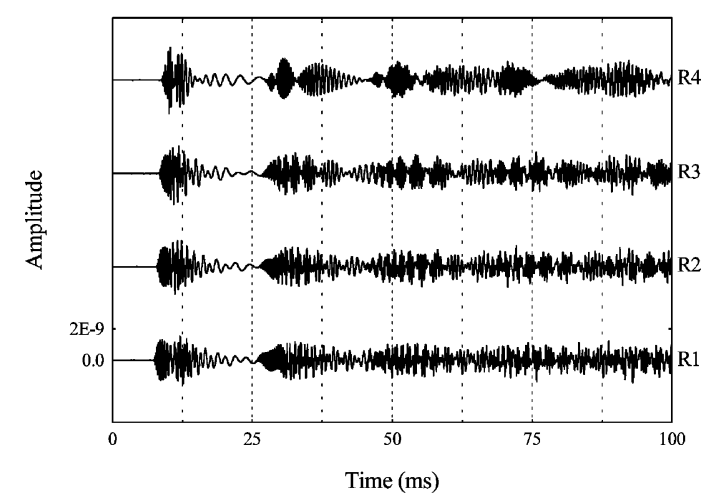

(b)

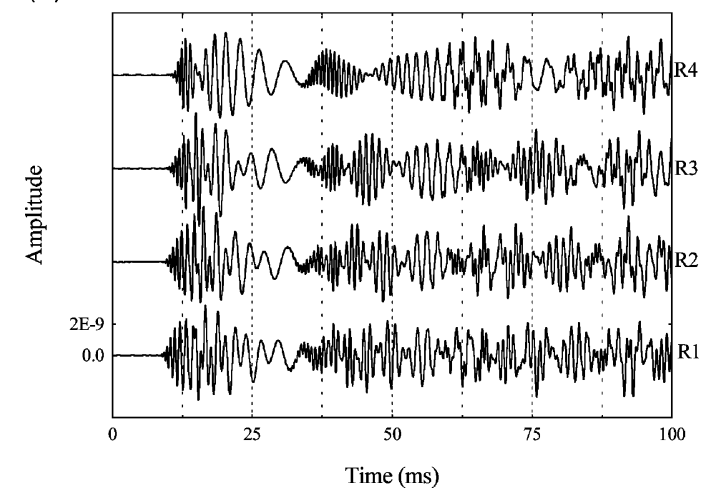

Fig. 9. Vertical time domain displacements obtained with the BEM model for the slab $0.10 \mathrm{~m}$ thick, with two lateral confinements, when a vertical line load is applied $\left(k_{z}=0 \mathrm{rad} / \mathrm{m}\right)$ : (a) Characteristic frequency of excitation $3000 \mathrm{~Hz}$; (b) Characteristic frequency of excitation $1000 \mathrm{~Hz}$. displacements when the excitation frequency is $1000 \mathrm{~Hz}$. The response exhibits similar trends to those observed in Fig. 9a but it retains the particular features relating to the low excitation frequency, that is lower velocities and higher amplitudes.

\section{Conclusions}

This paper describes the displacement field computed within slabs with and without lateral confinements. Analytical functions were used to obtain the three-dimensional displacement field within a slab of infinite extent. The same analytical functions have been incorporated as Green's functions in a BEM model to compute the wave field in a two-dimensional slab of finite and semi-infinite extent, avoiding the discretization of the free horizontal surfaces.

The results for the simulations reveal a wave field composed by body waves and dispersive surface guided waves. As the thickness of the layer increases the guided waves are less dispersive and become faster, particularly at low frequencies. The inclusion of lateral rigid boundaries in an infinite slab generates additional pulses due to the reflections on them and the enhancement of the amplitude responses, as observed in the time plots.

The waves propagating with an apparent wave velocity along the $z$ axis of $c=2600 \mathrm{~m} / \mathrm{s}$ reach the receivers at earlier times than those propagating at $c=\infty \mathrm{m} / \mathrm{s}$ $\left(k_{\mathrm{z}}=0 \mathrm{rad} / \mathrm{m}\right)$, revealing a reduction in amplitude.

\section{References}

[1] Graff KF. Wave motion in elastic solids. New York: Dover Publications, 1975.

[2] Tadeu A, António J. Green's functions for 2.5D elastodynamic problems in a free solid layer formation. Eng Struc 2002;24(4):491-9.

[3] Bouchon M. Discrete wave number representation of elastic wave fields in three-space dimensions. J Geophys Res 1979;84:360914.

[4] Kim J, Papageorgiou AS. Discrete wavenumber boundary element method for 3-D scattering problems. J Eng Mech ASCE 1993;119(3):603-24.

[5] Lamb H. On the propagation of tremors at the surface of an elastic solid. Phil Trans R Soc 1904;A203:1-42.

[6] Phinney RA. Theoretical calculation of the spectrum of first arrivals in layered elastic mediums. J. Geophys Res 1965;70:5107-23.

[7] Beskos DE. Boundary element methods in dynamic analysis: Part II (1986-1996). Appl Mech Rev 1997;50(3):149-97.

[8] Manolis GD, Beskos DE. Boundary element methods in elastodynamics. London: Unwin Hyman (sold to Chapman and Hall), 1988.

[9] Tadeu A, Santos PFA, Kausel E. Closed-form integration of singular terms for constant, linear and quadratic boundary elements-Part I: SH wave propagation. EABE -Engineering Analysis with Boundary Elements 1999;23(8):671-81.

[10] Tadeu A, Santos PFA, Kausel E. Closed-form integration of 
singular terms for constant, linear and quadratic boundary elements-Part II: SV-P wave propagation. EABE -Engineering Analysis with Boundary Elements 1999;23(9):757-68.

[11] Ricker N. The form and laws of propagation of seismic wavelets. Geophysics 1953;18:10-40.
[12] Kausel E, Roesset JM. Frequency domain analysis of undamped systems. J Eng Mech ASCE 1992;118(4):721-34. 